\title{
La protéomique quantitative par la méthode SILAC
}

\section{Technique et perspectives}

Anouk Emadali, Maighread Gallagher-Gambarelli

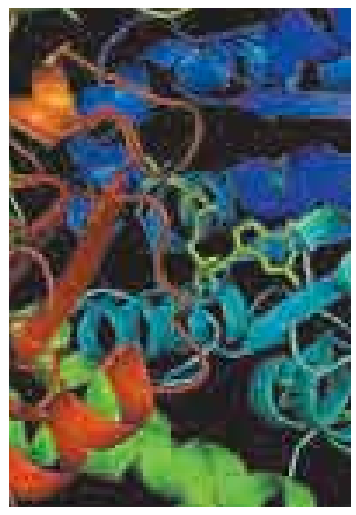
CEA, DSV, iRTSV, Laboratoire
d'étude de la dynamique
des protéomes, Grenoble,
F-38054, France;
Inserm, U880, Grenoble,
F-38054, France ;
Université Joseph Fourier,
17, rue des Martyrs,
38054 Grenoble, France.
maighread_gallagher@
hotmail.com
anouk.emadali@cea.fr

\section{La protéomique quantitative}

tées comme un seul échantillon, ce qui permet de préparer les protéines d'intérêt sans risquer d'introduire des erreurs de quantification. Au cours de l'analyse par spectrométrie de masse, l'abondance relative entre les échantillons biologiques peut être calculée pour chaque protéine en comparant l'intensité des peptides légers et lourds. Comme le montrent ses applications à diverses problématiques biologiques et cliniques, la méthode SILAC est particulièrement prometteuse pour l'élucidation des mécanismes moléculaires impliqués dans les grandes fonctions cellulaires, ainsi que pour l'identification de biomarqueurs de maladies. La limitation de la méthode SILAC à la quantification de protéines issues de cellules en culture vient d'être levée suite à la description d'une souris SILAC dont toutes les protéines sont marquées isotopiquement. Ces travaux laissent présager une extension de cette stratégie analytique à l'étude différentielle de tissus et de fluides biologiques d'animaux modèles. <

\section{Généralités}

Le domaine de la protéomique - définie comme l'étude de l'ensemble des protéines d'un échantillon biologique donné - s'est développé de façon spectaculaire au cours des dernières années $[1,2]$. Les progrès majeurs réalisés dans le domaine de l'instrumentation pour la protéomique (spectrométrie de masse, chromatographie liquide nano-débit), associés à la masse d'informations sans cesse croissante disponible dans les banques de données et à la conception de nouveaux outils logiciels, ont conduit à la mise en place de stratégies qui permettent d'analyser le protéome des échantillons biologiques de manière efficace et fiable. Ainsi, des inventaires de protéines de plus en plus complets ont pu être établis dans le cadre de travaux menés chez l'homme et chez des organismes modèles. Indéniablement, ces inventaires réalisés à partir d'échantillons plus ou moins complexes et ciblés (tissus, cellules, compartiments subcellulaires, complexes protéiques, etc.) ont participé à une meilleure connaissance des acteurs moléculaires impliqués dans les grands «mécanismes du vivant». Le principe général d'une analyse protéomique est présenté dans la Figure 1 et l'Encadré 1 . Les entités analysées par ce type d'approche sont les peptides issus de la digestion enzymatique des protéines.

Cependant, le protéome est une entité hautement dynamique. De ce fait, un enjeu majeur pour la 


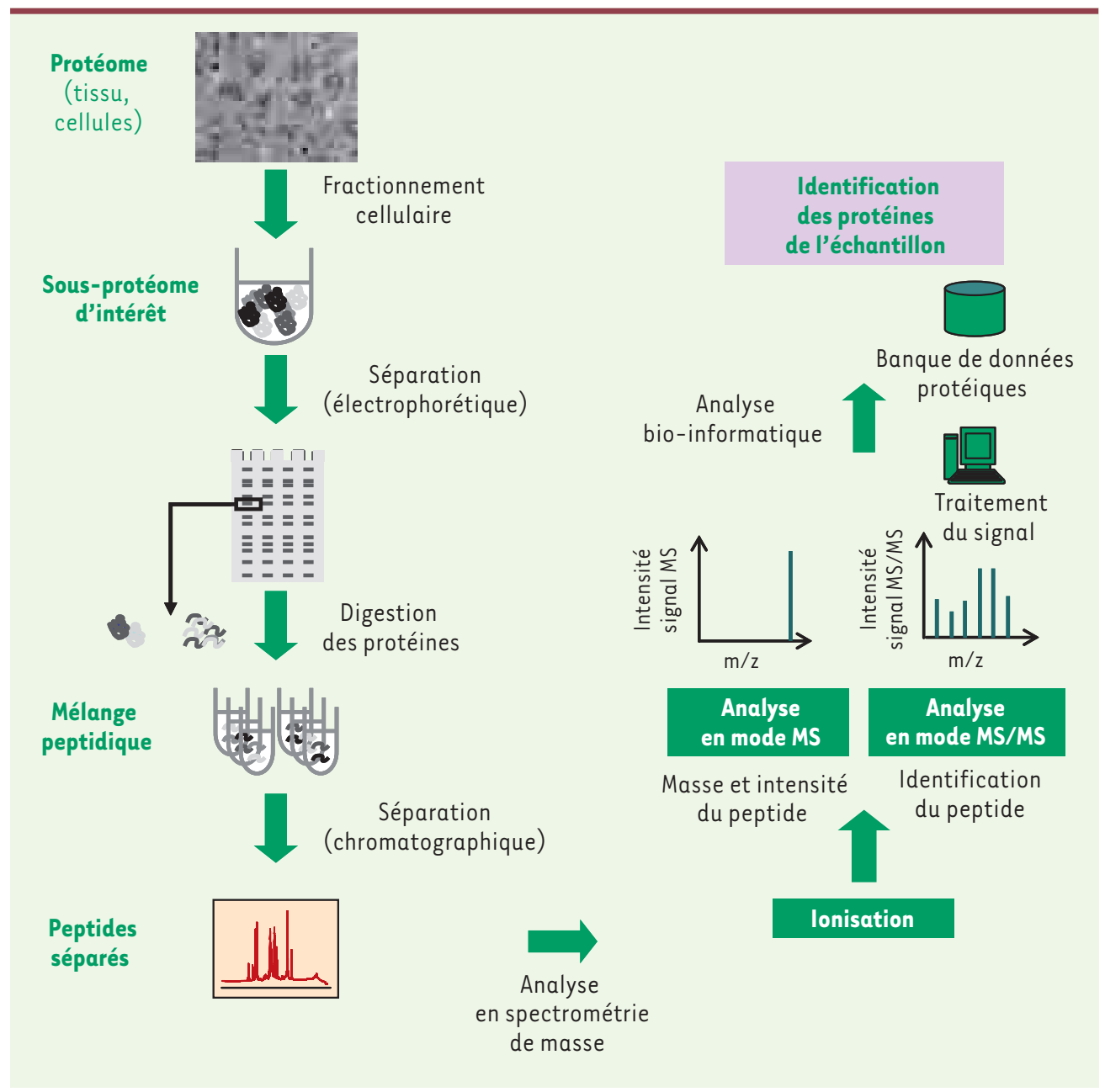

Figure 1. Déroulement d'une analyse protéomique basée sur la spectrométrie de masse. Une analyse protéomique classique se déroule comme suit: la première étape (à gauche) consiste généralement à fractionner l'échantillon pour le décomplexifier afin de rendre accessible à l'analyse des protéines peu abondantes ou de cibler un complexe protéique. Cette séparation peut être basée sur des méthodes biochimiques (fractionnement subcellulaire, colonnes d'affinité, copurification, etc.) et/ou électrophorétiques (SDS-PAGE ou 2D-GE). Les protéines ainsi séparées sont ensuite digérées par une enzyme protéolytique comme la trypsine. Les peptides générés sont séparés par chromatographie liquide, ionisés, analysés en mode MS afin d'en déduire leur masse (à droite). Les peptides sélectionnés sont fragmentés et analysés en mode MS/MS. Les spectres de fragmentation obtenus sont confrontés avec les spectres MS/

MS théoriques obtenus in silico pour chaque peptide trypsique prédit à partir des séquences répertoriées dans une banque de données protéique pertinente. Cette confrontation permet d'identifier la séquence en acides aminés du peptide qui a été fragmenté et, par conséquent, d'identifier la protéine correspondante.

protéomique consiste aujourd'hui à développer la protéomique quantitative et les outils et méthodes qui permettront d'appréhender cette nouvelle dimension de l'analyse. Le décryptage systématique des variations d'abondance du protéome en réponse à divers facteurs (hormone, stress, délétion de gène, pathologie, etc.) constitue en effet un moyen puissant pour approfondir notre connaissance des mécanismes cellulaires ou rechercher de nouveaux marqueurs de maladies. Ces techniques présentent un avantage sur les techniques de transcriptomique puisqu'elles s'intéressent aux protéines, les principaux acteurs du vivant et non à leurs précurseurs, les ARNm dont le niveau d'expression n'est pas toujours corrélé à l'abondance des protéines correspondantes.

\section{Les différentes stratégies d'analyse protéomique quantitative}

Il existe de plus en plus de méthodes de protéomique quantitative. Elles peuvent être classées en deux groupes : les techniques avec marquage et les techniques sans marquage. Ces différentes stratégies sont décrites dans des articles de revues $[3,4]$.
- Les techniques sans marquage

Elles incluent l'électrophorèse 2D (2D-GE) (GE pour gel electrophoresis) [5], la comparaison de traces MS (MS pour spectrométrie de masse) [6] et le spectral count (ou comptage de spectres MS/MS) [7] (Figure l et Encadré 1).

La 2D-GE est une méthode très résolutive qui permet de repérer les protéines qui sont différentiellement exprimées entre deux ou plusieurs échantillons. Cependant, cette technique affiche certaines limites: en particulier, elle s'applique très difficilement aux protéines membranaires et aux protéines présentant des points isoélectriques extrêmes. De plus, cette méthode est difficilement adaptable à une stratégie analytique «haut débit» et est limitée à l'étude des protéines les plus abondantes.

Avec le développement de spectromètres de masse couplés à des systèmes de chromatographie liquide, il est devenu possible d'analyser rapidement l'abondance des protéines issues d'échantillons biologiques complexes en comparant 


\section{Les différents modes d'analyse par spectrométrie de masse}

En complément de l'information donnée dans la Figure 1: l'analyse protéomique effectuée par spectrométrie de masse (MS) couplée à une chromatographie liquide haute performance à nano-débit (nano-LC pour nano-chromatographie liquide) se déroule en deux étapes. Brièvement, le mélange complexe de peptides issu de la digestion trypsique d'un échantillon est injecté sur une colonne nano-LC (phase-inverse). Les peptides sont séparés selon leur hydrophobicité, élués et injectés directement au niveau du spectromètre de masse pour analyse. Schématiquement, au niveau d'un spectromètre de masse, il y a deux modes d'analyse couplant la chromatographie liquide et l'analyse par spectrométrie de masse: le mode nano-LC-MS, qui fournit des informations sur l'abondance de chaque peptide trypsique (intensité du signal d'ion) présent dans l'échantillon, et le mode tandem ou nano-LC-MS/MS qui permet d'avoir accès à des informations sur la séquence en acides aminés des peptides. Ces deux modes d'analyse sont couramment utilisés dans les stratégies de recherche qui font appel à des méthodes de marquage isotopique.

directement les intensités de signal de chaque peptide entre différents échantillons. La comparaison de traces MS s'appuie sur la correspondance qui existe entre l'intensité du pic détecté par le spectromètre de masse en mode MS et la quantité de l'espèce correspondant à ce pic MS dans le mélange analysé. D'autre part, le comptage de spectres est une méthode récente qui permet de relier l'abondance d'une protéine au nombre de spectres MS/MS qui ont permis d'identifier cette protéine.

Toutes ces méthodes demandent une parfaite maîtrise et de la rigueur, à la fois dans les étapes de préparation des échantillons en amont et dans le traitement bio-informatique et statistique des données en aval de l'analyse en masse. Elles ont cependant l'avantage de pouvoir être appliquées à tout type d'échantillons biologiques, que ce soit des cellules en culture ou des échantillons cliniques (biopsies, fluides biologiques, etc.), de permettre la comparaison d'un grand nombre d'échantillons et de représenter un faible coût de mise en œuvre.

- Les techniques de protéomique comparative avec marquage

Elles sont nombreuses et variées. Basée sur la 2D-GE, la technique 2D-DIGE (differential in gel electrophoresis) [8] permet l'analyse sur un même gel de deux échantillons protéiques préalablement marqués avec des fluorophores distincts. Toutefois, aujourd'hui, la majorité des techniques de protéomique quantitative avec marquage s'appuient sur l'utilisation d'isotopes stables pour une analyse différentielle en spectrométrie de masse. Pour comparer deux échantillons, les protéines de l'un sont marquées avec des isotopes lourds (protéines «lourdes ») alors que les protéines du deuxième échantillon sont marquées avec des isotopes légers (protéines «légères »). L'analyse en spectrométrie de masse permet de repérer des paires de peptides lourds et légers dont l'abondance relative peut être calculée. Le marquage isotopique dans les échantillons à étudier peut se faire de différentes manières: marquage

Figure 2. Stratégies de protéomique quantitative et impact sur la précision de la quantification. Les échantillons à comparer sont représentés en bleu et rouge. La ligne pleine horizontale représente le stade où les échantillons sont combinés. Quand les échantillons sont traités en parallèle (lignes pointillées), des erreurs de quantification non compensées peuvent être introduites. SILAC: stable isotope labelling by amino acids in cell culture; ICAT: isotope coded affinity tags; ITRAP : isobaric tag for relative and absolute quantification (adapté de [3]).
Purification ou

fractionnement

Peptides

Échantillon analysé en spectrométrie de masse comptage de spectres

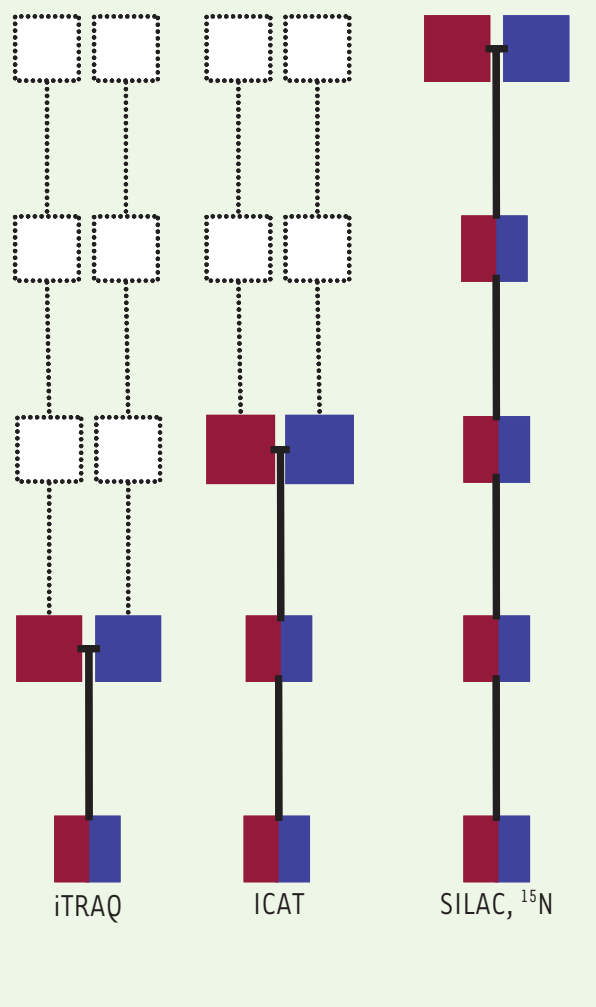

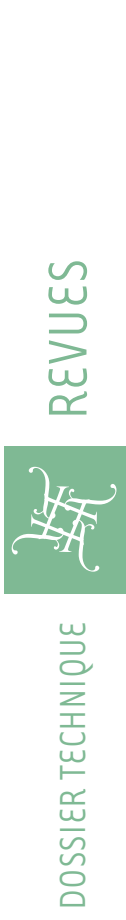




\section{Les acides aminés utilisés dans les approches SILAC}

Les choix de l'acide aminé à marquer et de la nature du marquage sont influencés par quelques règles:

1. L'acide aminé doit être de préférence un acide aminé essentiel (ce qui évite la fabrication de l'acide aminé léger par la cellule), ce qui explique d'ailleurs que le SILAC ne puisse être utilisé chez les cellules végétales qui sont autotrophes (capables d'utiliser des éléments inorganiques pour synthétiser leurs propres constituants organiques). De plus, cet acide aminé doit être présent dans la plupart des peptides. C'est pour ces raisons que les premiers essais de type SILAC ont été faits avec la leucine (Leu) marquée [12, 20]. L'acide aminé essentiel le plus abondant est la Leu (présent dans plus de $50 \%$ des peptides trypsiques). Le marquage par le deutérium étant relativement peu coûteux, ces premières études ont utilisé des isotopes deutérés de Leu (respectivement ${ }^{2} \mathrm{H}_{10}$-Leu ou ${ }^{2} \mathrm{H}_{3}$-Leu). Par la suite, des isotopes marqués ${ }^{13} \mathrm{C}$ et/ou ${ }^{15} \mathrm{~N}$ d'arginine (Arg) et de lysine (Lys) ont été préférés $[25,41]$. L'Arg n'est pas strictement un acide aminé essentiel, mais il est essentiel pour la survie des cellules en culture [42, 43]. L'utilisation d'un double marquage Arg, Lys en combinaison avec la digestion trypsique offre l'avantage d'avoir un simple marquage sur presque tous les peptides - la trypsine clivant spécifiquement les protéines en carboxy-terminal d'un résidu Arg ou Lys. Seuls les peptides carboxy-terminaux des protéines peuvent ne pas avoir de marquage, et les quelques peptides ayant échappé au clivage auront un double marquage. Le marquage de presque tous les peptides permet une quantification plus précise de plus de protéines.

2. Le marquage ne doit pas changer les propriétés physicochimiques des protéines ou peptides. La précision de la quantification par spectrométrie de masse dépend de plusieurs facteurs : l'ionisation des peptides, l'état des détecteurs et l'environnement des peptides. Le remplacement d'un atome d'hydrogène par un atome de deutérium n'est pas sans influence sur le comportement des peptides sur la colonne de chromatographie en phase inverse. Dans les premières études SILAC, les «paires » de peptides lourds et légers se trouvaient séparées au cours de l'étape nanoLC avant injection dans le spectromètre de masse, ce qui compliquait leur détection et pouvait fausser leur quantification $[12,20]$. Le remplacement d'un atome de ${ }^{12} \mathrm{C}$ par un ${ }^{13} \mathrm{C}$ ou d'un ${ }^{14} \mathrm{~N}$ par un ${ }^{15} \mathrm{~N}$ semble être plus neutre. Les paires de peptides coéluent et peuvent être quantifiées plus aisément.

3. L'acide aminé marqué ne doit pas être métabolisé par la cellule. En effet, si l'acide aminé est métabolisé par la cellule, le marquage sera redistribué sur les acides aminés nouvellement synthétisés, ce qui complique les spectres et peut induire des erreurs de quantification. Pour les cellules humaines, I'utilisation de l'Arg marqué peut induire ce type de redistribution du marquage (conversion d'Arg en proline [Pro]) [25, 44-47]. Une approche permet de minimiser l'incidence de cette conversion sur les résultats de quantification, elle consiste à marquer les deux échantillons à comparer avec deux isotopes différents d'Arg. Le taux de conversion devrait être le même dans les deux lots de cellules. Alternativement, deux approches permettent d'éviter la conversion d'Arg en Pro: la première consiste à réduire la quantité d'Arg rajoutée au milieu de culture, mais certaines lignées cellulaires ne supportent pas un milieu pauvre en Arg [47] ; la deuxième consiste à ajouter de la Pro en excès dans le milieu [48], ce qui semble convenir à la plupart des lignées cellulaires.

4. Selon l'application, certains acides aminés présentent un intérêt particulier. Pour l'étude de voies de signalisation, un marquage utilisant des isotopes de Tyr a été utilisé [49]. métabolique [9-12], marquage chimique (ICAT ou isotope coded affinity tags, iTRAP ou isobaric tag for relative and absolute quantification) [13-15], marquage enzymatique [16] ou marquage postenzymatique $[17,18]$.

Chaque méthode présente ses avantages et ses inconvénients. Toutefois, il est très important d'introduire l'étape de marquage isotopique le plus précocement possible dans le processus de préparation des échantillons. Ceci permet en effet de limiter les erreurs de quantification inéluctables liées à la manipulation en parallèle des échantillons à comparer (Figure 2). Cette étape de marquage précoce peut passer par un marquage métabolique des protéines comme c'est le cas pour le marquage ${ }^{15} \mathrm{~N}$ et pour la technique SILAC (stable isotope labelling by amino acids in cell culture).

Le traitement informatique des données issues de marquages métaboliques au ${ }^{15} \mathrm{~N}$ est délicat car l'écart de masse introduit par le marquage entre un peptide léger et un peptide lourd est fonction du nombre d'azotes présents dans le peptide alors qu'il est fixe dans le cas du SILAC. Ceci rend les données plus faciles à traiter. Ainsi, à l'heure actuelle, la stratégie SILAC est l'une des méthodes de protéomique quantitative les plus fiables et les plus performantes. Ces atouts sont d'ailleurs reflétés par la bibliométrie qui révèle que, comparativement aux autres techniques de protéomique quantitative avec marquage, on a observé au cours des six dernières années un véritable «décollage » du nombre d'articles utilisant le SILAC. D'un seul article en 2002 nous sommes passés à une soixantaine en 2008. Cette supériorité de la technique SILAC est d'autant plus flagrante si l'on compare les facteurs d'impact des journaux dans lesquels ces articles sont publiés.

\section{La stratégie SILAC}

\section{Principe}

La méthode qui est maintenant connue sous le nom de SILAC avait d'abord été nommée AACT (amino acid coded mass tagging) $[10,11]$. Elle s'applique à des cellules maintenues en culture. Récemment, elle a été adaptée au marquage des tissus et organes d'une souris [19]. Le principe est schématisé dans la Figure 3.

- La première phase de l'expérience, la phase d'adaptation, consiste à cultiver une population de cellules en présence d'acides aminés isotopiquement alourdis jusqu'à ce que toutes les protéines aient incorporé ces acides aminés lourds dans une proportion comprise entre 95 et $100 \%$. Il est nécessaire de déterminer expérimentalement ces conditions d'incorporation pour chaque lignée ou type cellulaire. Les paramètres qui orientent le choix des acides aminés marqués 
A Phase d'adaptation

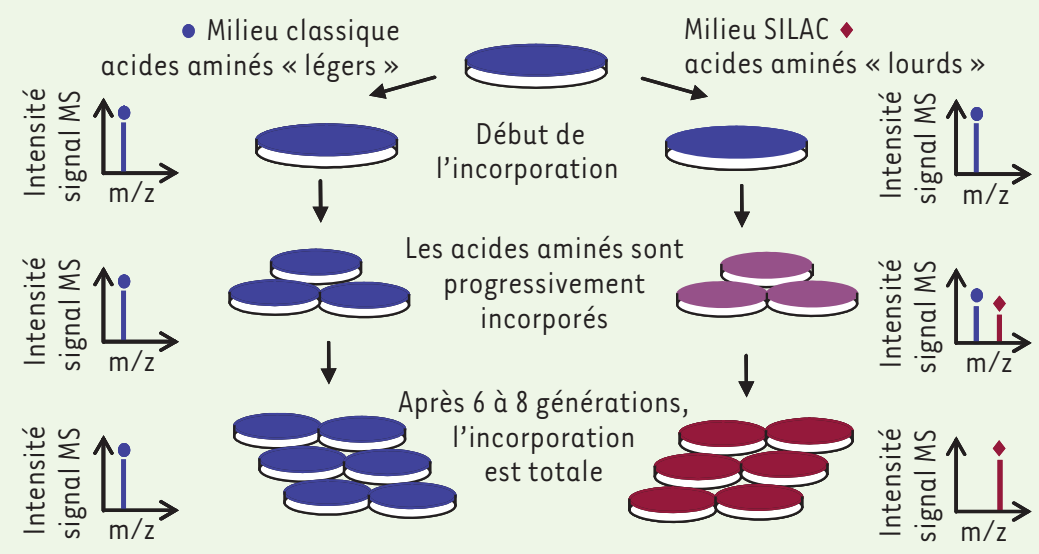

B Expérience
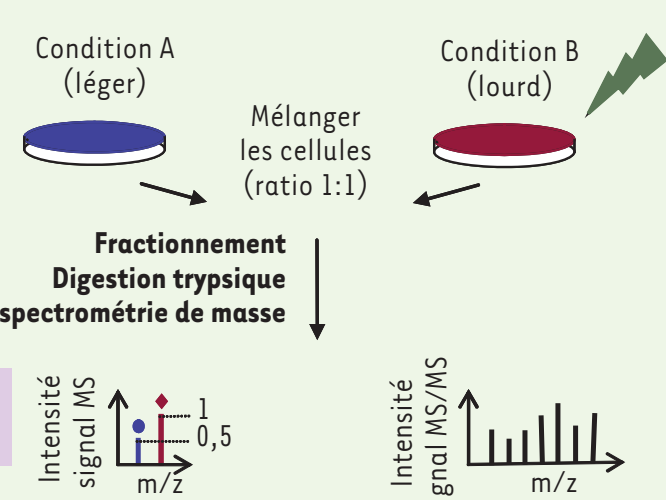

Stimulus, stress,... les cellules (ratio $1: 1$ )

\section{Fractionnement trypsique Analyse en spectrométrie de masse}

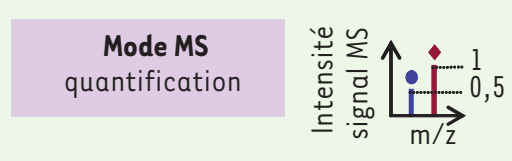

L'intensité des signaux MS des peptides lourds et légers permet de calculer l'abondance relative des protéines correspondantes (ici le peptide est 2 fois moins abondant dans la condition A que dans la condition $B$ )
Figure 3. Représentation schématique de la stratégie SILAC. Une expérience SILAC se compose de deux phases distinctes: une phase d'adaptation ( $A$ ) et une phase expérimentale (B). A. Durant la phase d'adaptation, les cellules sont cultivées dans un milieu où certains acides aminés ont été substitués par leurs isotopes «lourds » jusqu'à ce que les cellules aient complètement incorporé les acides aminés marqués (losange rouge sur les graphes et couleur des boîtes). En parallèle, une culture en milieu standard est effectuée. $B$. Dans la seconde phase, l'expérience en tant que telle, suite à l'incorporation du marquage et à l'application d'un stress ou stimulus à une des populations cellulaires, les lots de cellules à comparer sont mélangés. L'échantillon total ou un sous-protéome est analysé par spectrométrie de masse. Les peptides lourds et légers, qui peuvent être distingués grâce au marquage isotopique différentiel (point bleu versus losange rouge sur les graphes), seront quantifiés en mode MS et identifiés en mode MS/MS (adapté de [3]). sont discutés dans l'Encadré 2. II a été démontré à plusieurs reprises que les milieux de culture modifiés n'affectaient en rien la croissance, division, morphologie ou réponse biologique des cellules [12, 20]. Généralement, après 6 à 8 temps de doublement de la population cellulaire, toutes les protéines ont incorporé les acides aminés alourdis avec un taux d'incorporation proche de $100 \%$. II a récemment été démontré que la méthode SILAC n'était pas restreinte aux cellules qui prolifèrent en culture, mais pouvait être appliquée à des cellules neuronales primaires $[21,22]$. Dans le cas de «souris SILAC», le marquage total de toutes les protéines a été obtenu au cours de la deuxième génération de souris nourries avec de la lysine marquée ${ }^{13} \mathrm{C}[19]$.

Dans toutes les expériences de type SILAC, I'incorporation des acides aminés peut être suivie par spectrométrie de masse en mode MS. Au cours du marquage, pour chaque peptide, le peptide lourd apparaît sur la trace MS séparé du peptide léger par un écart correspondant à la différence de masse entre les acides aminés légers et lourds. L'incorporation est considérée comme totale lorsque les pics correspondant aux peptides légers ne peuvent plus être détectés sur la trace MS.
Les protéines lourdes se trouvent alors comme «marquées au fer rouge $\gg$.

- Au cours de la phase expérimentale, après avoir cultivé une population cellulaire dans un milieu normal et l'autre dans un milieu isotopiquement alourdi pendant le temps nécessaire au marquage, un stress ou stimulus peut être appliqué à l'une des populations. Les cellules issues des deux conditions de culture sont alors mélangées dans un rapport 1:1. Les échantillons peuvent ensuite être fractionnés, les protéines digérées et les peptides analysés en MS et MS/MS. L'information concernant l'origine de chaque protéine est conservée grâce à l'écart de masse introduit par le marquage isotopique qui est facilement détectable au moment de l'analyse protéomique. Chaque peptide est donc détecté par le spectromètre de masse sous la forme d'une paire constituée d'une forme lourde et d'une forme légère. L'analyse protéomique comparative consiste alors à repérer les paires sur la trace MS. Pour chaque paire, le rapport des intensités du signal des formes lourdes et légères 
traduit la différence de concentration de ces peptides dans le mélange analysé. En corrélant données MS et données MS/MS, il est possible de remonter à l'identification des peptides et donc des protéines qui présentent des différences d'abondance entre les échantillons à comparer.

\section{Acquisition, traitement et validation}

des données de spectrométrie de masse

II n'existe pas de spectromètre de masse particulièrement dédié à l'analyse des échantillons SILAC. Cependant, les spectromètres de masse de nouvelle génération, qui offrent une grande précision de masse, une haute résolution et une vitesse d'acquisition élevée des données en mode MS/MS, permettent d'identifier et de quantifier un plus grand nombre d'espèces peptidiques.

La partie la plus délicate de la stratégie SILAC consiste à analyser les données informatiques avec des outils dédiés et performants qui (1) détectent les espèces présentes sous forme de paires sur la trace MS, (2) calculent les ratios d'intensité pour chaque paire et (3) font correspondre à chaque paire une séquence obtenue grâce à l'analyse MS/MS. De nombreux outils logiciels ont été développés pour effectuer une ou plusieurs de ces étapes. Deux de ces outils semblent particulièrement prometteurs: MFPaP [23] et MaxQuant [24]. Ils présentent l'intérêt d'être des logiciels libres (open source). Bien que restreints aux données issues d'un type de configuration instrumentale, ces deux outils se démarquent par leur relative facilité d'utilisation. De ce fait, la technique SILAC pourrait, dans un futur proche, être accessible à un public de plus en plus large. Au vu du nombre de protéines différentiellement exprimées et identifiées, le problème de la validation des résultats se pose pour les résultats d'analyses SILAC, tout comme pour les autres méthodes haut débit, qu'elles soient transcriptomiques ou protéomiques. L'analyse statistique de données issues de ce type d'analyse peut faciliter l'acceptation d'un résultat par la communauté mais ne peut pas remplacer la validation par des méthodes complémentaires qui est indispensable pour confirmer la différence d'expression mesurée.

\section{Applications}

La méthode SILAC a été utilisée dans de nombreuses études utilisant divers modèles biologiques dont les applications étaient fondamentales ou cliniques.

\section{- Dynamique de voies de signalisation cellulaire}

$\mathrm{Si}$ les premières études se sont intéressées à la différenciation de cellules souches [12, 25], l'essentiel de la littérature utilisant le SILAC concerne l'étude de la dynamique de voies de signalisation intracellulaires via l'étude de modifications post-traductionnelles, notamment de phosphorylations et de méthylations. Les articles les plus marquants se sont intéressés à l'étude des voies de signalisation activées par l'EGF (epidermal growth factor) dans les cellules mammifères $[26,27]$ ou par les phéromones chez la levure [28, 29].

\section{- Reconstitution de réseaux d'interaction}

L'utilisation du SILAC pour étudier les réseaux protéiques est en pleine expansion, comme le reflètent les nombreux articles décrivant cette application. En effet, en réalisant une comparaison entre une expérience de coprécipitation et un contrôle négatif, le SILAC permet de discriminer les vraies interactions et les faux positifs et ainsi d'évaluer la spécificité d'interactions protéiques [3032]. Un autre aspect particulièrement intéressant de cette application est qu'elle permet de distinguer les partenaires labiles des partenaires stables d'un complexe protéique [33] et représente ainsi une stratégie originale et élégante pour aborder la dynamique des complexes protéiques. Récemment, l'utilisation du SILAC pour étudier les partenaires d'interaction a été étendue à l'étude de complexes ADN/protéine [34].

\section{- Protéomique spatiotemporelle}

Un autre aspect intéressant de la technique SILAC est la possibilité qu'elle offre d'approfondir l'analyse protéomique en étudiant spécifiquement l'évolution du contenu protéique ou le taux de renouvellement des protéines d'un sous-protéome d'intérêt (complexe protéique ou compartiment cellulaire). Bien que d'autres techniques de protéomique quantitative permettent également d'atteindre ce but, la technique SILAC est particulièrement adaptée à ce type d'études fonctionnelles, ne serait-ce que parce qu'elle permet de mélanger les échantillons à comparer à un stade précoce du traitement et ainsi d'éviter des erreurs de quantification dues à la préparation en parallèle des échantillons biologiques. À titre d'exemple on peut citer l'étude de l'effet de l'actinomycine (un inhibiteur de la transcription) sur la dynamique des protéines nucléolaires des cellules Hela [35] et le taux de renouvellement des protéines du $\mathrm{CMH}$ (complexe majeur d'histocompatibilité) dans une lignée tumorale ovarienne [36].

\section{- Identification de biomarqueurs pathologiques}

D'un point de vue clinique, le SILAC est une méthode de choix pour identifier des marqueurs de maladies à partir de lignées cellulaires modèles. Il a par exemple été utilisé avec succès pour identifier des biomarqueurs spécifiques de cellules cancéreuses en comparant lignées de cellules pancréatiques [37] et mammaires [38] normales et tumorales. Le SILAC a également été utilisé pour évaluer des réponses pharmacologiques comme celle déclenchée par l'inhibition du récepteur ERBB2 (ou HER2/neu, human epidermal growth factor receptor 2) dans le traitement de certains cancers du sein [39]. II est toutefois important de rappeler que l'utilisation du SILAC dans le contexte d'une recherche de biomarqueurs implique l'utilisation de lignées cellulaires modèles réellement pertinentes 
pour l'étude de la pathologie en question. De plus, nous voudrions insister sur le fait que l'utilisation du SILAC est restreinte à la phase d'identification de ces marqueurs à partir de cellules en culture. $\varepsilon$ n dehors du fait que le SILAC in vivo ne peut être étendu à l'homme, les techniques de protéomique sans marquage sont plus adaptées pour l'identification et surtout pour la validation de biomarqueurs impliquant le criblage de nombreux échantillons cliniques. Le problème de la validation des résultats est d'ailleurs particulièrement sensible pour les projets qui visent à identifier des marqueurs de maladies, quelle que soit la stratégie utilisée. Ainsi, un grand nombre de candidats biomarqueurs ont été identifiés par des techniques de protéomique quantitative mais ces candidats prometteurs n'ont pas trouvé d'application en clinique, par manque de stratégies de validation haut débit [40].

- Vers des études in vivo

Une nouvelle étape dans le développement et les applications potentielles du SILAC a récemment été franchie avec l'obtention d'une souris SILAC dont toutes les protéines ont incorporé des isotopes ${ }^{13} \mathrm{C}$ de lysine [19]. Cette étude a permis de déterminer que le marquage n'a aucun effet sur la croissance, la reproduction ou le comportement des souris. En comparant souris knock out et sauvages, les effets de l'inactivation de trois protéines impliquées dans la formation du complexe d'adhésion cellulaire médié par les intégrines ont pu être étudiés. Les auteurs ont ainsi pu identifier des protéines impliquées dans la structure de la membrane plasmique des érythrocytes dont le niveau d'expression est modifié chez la souris en l'absence d'expression de la protéine Kindlin. Ces modifications du protéome sont corrélées avec l'anémie sévère qui caractérise le phénotype observé chez cette souris.

Cette souris est donc un outil extrêmement prometteur pour étudier de manière quantitative les protéomes d'organes et de fluides biologiques et pour déterminer les mécanismes moléculaires liés aux phénotypes observés in vivo.

\section{Conclusion}

Bien que le marquage métabolique à l'échelle d'un animal entier en soit encore à ses balbutiements, les nombreuses applications du SILAC permettent de prendre la mesure du potentiel que représente le développement de cette technique. II est probable que, dans un futur proche, l'approche SILAC soit encore essentiellement utilisée dans le domaine de l'étude des cellules en culture, ne serait-ce que pour des raisons de coût. En effet, les milieux de culture adaptés au marquage SILAC étant déjà relativement onéreux, il est facile d'imaginer le coût extrêmement élevé du marquage d'un animal entier. Malgré cet obstacle, partagé par toutes les méthodes de protéomique quantitative impliquant un marquage isotopique, le SILAC est une méthode de choix pour la réalisation d'analyses quantitatives fiables, robustes et reproductibles.

Le développement d'une nouvelle génération d'outils bio-informatiques plus conviviaux dédiés à l'exploitation des résultats d'expériences
SILAC incite à penser que cette technique est amenée à poursuivre son développement et à être de plus en plus largement utilisée. Combinée à des approches traditionnelles de biologie cellulaire et de biochimie, la méthode SILAC est amenée à devenir, dans un futur proche, une stratégie de choix pour aborder l'étude de nombreuses problématiques en biologie. $\diamond$

\section{SUMMARY}

Quantitative proteomics by SILAC:

practicalities and perspectives

for an evolving approach

Mass spectrometry-based quantitative proteomics strategies are ideally adapted to the detection of global protein changes between different biological samples. Among these, SILAC (stable isotope labelling by amino acids in cell culture) has demonstrated a great potential. This method is extremely accurate and relatively easy to apply for the quantification of proteins extracted from cultured cells. SILAC involves cell culture either in regular culture media ("light" protein synthesis) or in media where amino acids have been replaced by their isotopically labelled counterparts ("heavy" protein synthesis). Cell populations to be compared can be mixed and treated as a single sample, which allows downstream sample preparation without the risk of introducing quantification errors. During mass spectrometry analysis, the relative protein abundance between biological samples can be calculated from the intensities of heavy and light peptides. As shown by numerous applications in biological and clinical studies, SILAC represents a promising method for the elucidation of cellular and physio-pathological mechanisms and for the identification of disease biomarkers. The restriction of SILAC to the quantification of proteins from cultured cells has just been overcome with the description in a recent paper of a SILAC mouse, which will allow this technology to be applied to the differential study of tissues and biological fluids from model animals. $\diamond$

\section{REMERCIEMENTS}

Les auteurs remercient Jérôme Garin et les autres membres du Laboratoire d'Étude de la Dynamique des Protéomes pour leurs commentaires constructifs sur cette revue. Anouk Emadali est bénéficiaire d'un financement post-doctoral INCa; le travail de Maighread Gallagher-Gambarelli est financé par un contrat Jeunes Chercheurs Inserm.

\section{CONFLIT D'INTÉRÊTS}

Les auteurs déclarent n'avoir aucun conflit d'intérêts concernant les données publiées dans cet article. 


\section{RéFÉRENCES}

1. Lescuyer $P$, Chevallet $M$, Rabilloud T. L'analyse protéomique : concepts, réalités et perspectives en thérapeutique. Med Sci (Paris) $2004 ; 20: 587-92$.

2. Solassol J, Boulle N, Maudelonde T, Mange A. Protéomique clinique : vers la détection précoce des cancers? Med Sci (Paris) $2005 ; 21: 722-8$

3. Ong SE, Mann M. Mass spectrometry-based proteomics turns quantitative. Nat Chem Biol $2005 ; 1: 252-62$.

4. Bantscheff M, Schirle M, Sweetman G, et al. Quantitative mass spectrometry in proteomics: a critical review. Anal Bioanal Chem 2007 ; 389 : 1017-31.

5. Gorg A, Weiss W, Dunn MJ. Current two-dimensional electrophoresis technology for proteomics. Proteomics $2004 ; 4: 3665-85$.

6. Wang M, You J, Bemis KG, et al. Label-free mass spectrometry-based protein quantification technologies in proteomic analysis. Brief Funct Genomic Proteomic $2008 ; 7: 329-39$.

7. Liu H, Sadygov RG, Yates JR. A model for random sampling and estimation of relative protein abundance in shotgun proteomics. Anal Chem $2004 ; 76: 4193-201$.

8. Alban A, David SO, Bjorkesten L, et al. A novel experimental design for comparative twodimensional gel analysis: two-dimensional difference gel electrophoresis incorporating a pooled internal standard. Proteomics $2003 ; 3: 36-44$

9. Lanquar V, Kuhn L, Lelievre F, et al. 15N-metabolic labeling for comparative plasma membrane proteomics in Arabidopsis cells. Proteomics $2007 ; 7: 750-4$.

10. Oda Y, Huang K, Cross FR, et al. Accurate quantitation of protein expression and site-specific phosphorylation. Proc Natl Acad Sci USA $1999 ; 96: 6591-6$

11. Chen $X$, Smith LM, Bradbury $E M$. Site-specific mass tagging with stable isotopes in proteins for accurate and efficient protein identification. Anal Chem $2000 ; 72: 1134-43$.

12. Ong SE, Blagoev $B$, Kratchmarova I, et al. Stable isotope labeling by amino acids in cell culture, SILAC, as a simple and accurate approach to expression proteomics. Mol Cell Proteomics 2002; $1: 376-86$.

13. Gygi SP, Rist B, Gerber SA, et al. Quantitative analysis of complex protein mixtures using isotope-coded affinity tags. Nat Biotechnol 1999; $17: 994-9$.

14. Ramus C, Gonzalez de Peredo A, Dahout C, et al. An optimized strategy for ICAT quantification of membrane proteins. Mol Cell Proteomics $2006 ; 5: 68-78$

15. Ross PL, Huang YN, Marchese JN, et al. Multiplexed protein quantitation in Saccharomyces cerevisiae using amine-reactive isobaric tagging reagents. Mol Cell Proteomics 2004 ; 3: 1154-69.

16. Yao X, Freas A, Ramirez J, et al. Proteolytic 180 labeling for comparative proteomics: model studies with two serotypes of adenovirus. Anal Chem $2001 ; 73: 2836-42$.

17. Munchbach M, Quadroni M, Miotto G, James P. Quantitation and facilitated de novo sequencing of proteins by isotopic $\mathrm{N}$-terminal labeling of peptides with a fragmentationdirecting moiety. Anal Chem $2000 ; 72$ : 4047-57.

18. Goodlett DR, Keller A, Watts JD, et al. Differential stable isotope labeling of peptides for quantitation and de novo sequence derivation. Rapid Commun Mass Spectrom 2001 ; $15: 1214-21$.

19. Kruger M, Moser M, Ussar S, et al. SILAC mouse for quantitative proteomics uncovers kindlin-3 as an essential factor for red blood cell function. Cell 2008; $134: 353-64$.

20. Jiang $H$, English AM. Quantitative analysis of the yeast proteome by incorporation of isotopically labeled leucine. J Proteome Res $2002 ; 1: 345-50$.

21. Spellman DS, Deinhardt K, Darie CC, et al. Stable isotopic labeling by amino acids in cultured primary neurons: application to brain-derived neurotrophic factor-dependent phosphotyrosine-associated signaling. Mol Cell Proteomics 2008 ; 7 : 1067-76.

22. Liao L, Park SK, Xu T, et al. Quantitative proteomic analysis of primary neurons reveals diverse changes in synaptic protein content in fmrl knockout mice. Proc Natl Acad Sci USA 2008 ; $105:$ 15281-6.

23. Bouyssie D, Gonzalez de Peredo A, Mouton $\varepsilon$, et al. Mascot file parsing and quantification (MFPaO), a new software to parse, validate, and quantify proteomics data generated by ICAT and SILAC mass spectrometric analyses: application to the proteomics study of membrane proteins from primary human endothelial cells. Mol Cell Proteomics 2007; 6: 1621-37.

24. Cox J, Mann M. MaxQuant enables high peptide identification rates, individualized p.p.b.-range mass accuracies and proteome-wide protein quantification. Nat Biotechnol $2008 ; 26: 1367-72$

25. Ong SE, Kratchmarova I, Mann M. Properties of 13 C-substituted arginine in stable isotope labeling by amino acids in cell culture (SILAC). J Proteome Res $2003 ; 2: 173-81$.

26. Blagoev B, Ong SE, Kratchmarova I, Mann M. Temporal analysis of phosphotyrosine-dependent signaling networks by quantitative proteomics. Nat Biotechnol $2004 ; 22$ : 1139-45.

27. Olsen JV, Blagoev B, Gnad F, et al. Global, in vivo, and site-specific phosphorylation dynamics in signaling networks. Cell $2006 ; 127: 635-48$.

28. Gruhler A, Olsen JV, Mohammed S, et al. Quantitative phosphoproteomics applied to the yeast pheromone signaling pathway. Mol Cell Proteomics 2005; $4: 310-27$
29. De Godoy LM, Olsen JV, Cox J, et al. Comprehensive mass-spectrometrybased proteome quantification of haploid versus diploid yeast. Nature $2008 ; 455: 1251-4$

30. De Hoog CL, Foster LJ, Mann M. RNA and RNA binding proteins participate in early stages of cell spreading through spreading initiation centers. Cell $2004 ; 117: 649-62$

31. Blagoev B, Kratchmarova I, Ong SE, et al. A proteomics strategy to elucidate functional protein-protein interactions applied to $\varepsilon G F$ signaling. Nat Biotechnol 2003; $21: 315-8$.

32. Vermeulen M, Mulder KW, Denissov S, et al. Selective anchoring of TFIID to nucleosomes by trimethylation of histone $\mathrm{H3}$ lysine 4. Cell 2007 ; $131: 58-69$.

33. Wang $X$, Huang L. Identifying dynamic interactors of protein complexes by quantitative mass spectrometry. Mol Cell Proteomics $2008 ; 7: 46-57$.

34. Mittler G, Butter F, Mann M. A SILAC-based DNA protein interaction screen that identifies candidate binding proteins to functional DNA elements. Genome Res 2009; 19: 284-93.

35. Andersen JS, Lam YW, Leung AK, et al. Nucleolar proteome dynamics. Nature $2005 ; 433: 77-83$

36. Milner $\varepsilon$, Barnea $\varepsilon$, Beer I, Admon A. The turnover kinetics of major histocompatibility complex peptides of human cancer cells. Mol Cell Proteomics $2006 ; 5: 357-65$.

37. Gronborg M, Kristiansen TZ, Iwahori A, et al. Biomarker discovery from pancreatic cancer secretome using a differential proteomic approach. Mol Cell Proteomics 2006 ; 5 : 157-71.

38. Liang X, Zhao J, Hajivandi M, et al. Quantification of membrane and membrane-bound proteins in normal and malignant breast cancer cells isolated from the same patient with primary breast carcinoma. J Proteome Res 2006 ; 5 : 2632-41.

39. Bose R, Molina H, Patterson AS, et al. Phosphoproteomic analysis of Her2/neu signaling and inhibition. Proc Natl Acad Sci USA 2006 ; $103: 9773-8$

40. Rifai N, Gillette MA, Carr SA. Protein biomarker discovery and validation: the long and uncertain path to clinical utility. Nat Biotechnol 2006 ; $24: 971-83$.

41. Ibarrola N, Kalume DE, Gronborg M, et al. A proteomic approach for quantitation of phosphorylation using stable isotope labeling in cell culture. Anal Chem 2003; $75: 6043-9$.

42. Scott L, Lamb J, Smith S, Wheatley DN. Single amino acid (arginine) deprivation: rapid and selective death of cultured transformed and malignant cells. BrJ Cancer $2000 ; 83: 800-10$.

43. Wheatley DN, Scott L, Lamb J, Smith S. Single amino acid (arginine) restriction: growth and death of cultured HeLa and human diploid fibroblasts. Cell Physiol Biochem 2000; $10: 37-55$.

44. Hwang SI, Lundgren DH, Mayya V, et al. Systematic characterization of nuclear proteome during apoptosis: a quantitative proteomic study by differential extraction and stable isotope labeling. Mol Cell Proteomics $2006 ; 5: 1131-45$.

45. Ong SE, Mann M. A practical recipe for stable isotope labeling by amino acids in cell culture (SILAC). Nat Protoc 2006; $1: 2650-60$

46. Schmidt F, Strozynski M, Salus SS, et al. Rapid determination of amino acid incorporation by stable isotope labeling with amino acids in cell culture (SILAC). Rapid Commun Mass Spectrom 2007 ; 21 : 3919-26.

47. Van Hoof D, Pinkse MW, Oostwaard DW, et al. An experimental correction for arginine-to-proline conversion artifacts in SILAC-based quantitative proteomics. Nat Methods $2007 ; 4$ : 677-8.

48. Bendall SC, Hughes $C$, Stewart MH, et al. Prevention of amino acid conversion in SILAC experiments with embryonic stem cells. Mol Cell Proteomics $2008 ; 7$ : 1587-97.

49. Amanchy R, Kalume DE, Iwahori A, et al. Phosphoproteome analysis of HeLa cells using stable isotope labeling with amino acids in cell culture (SILAC). J Proteome Res 2005 ; 4 : 1661-71.

\section{TIRÉS À PART}

M. Gallagher-Gambarelli 